\title{
Solar Tracking System with Momentary Tracking Based on Operational Amplifiers in Order to be Used in Photovoltaic Panels for Following the Sun
}

\author{
Sobhan AVARAND ${ }^{1}$, Mostafa PIRMORADIAN ${ }^{2 *}$
}

1- Department of Mechanical Engineering, Khomeishahr Branch, Islamic Azad University, Khomeinishahr/Isfahan, Iran

2- Department of Mechanical Engineering, Khomeishahr Branch, Islamic Azad University, Khomeinishahr/Isfahan, Iran

\begin{abstract}
This tracking system was designed based on the work of operational amplifiers and achieved maximum accuracy by two separate methods and was evaluated using op-amps specifications and comparing for twice the voltage of optical resistors which work is sensing. Optimization numerical results and achieved experiences showed good condition of this type of design. Angled and pyramid layout are used to get sunlight of these resistors; this created angle between the two resistors will raise the navigation accuracy as much as possible. If solar radiation reach the photovoltaic panel with deviation, it can reduce the output efficiency up to $50 \%$ and even more. A moment tracking is considered for this system in order to minimize this problem. In this work, it has been tried to prevent the complexity of electronic circuit and also equipment as much as possible. It will reduce the system costs in implementation and construction by raising efficiency and speed of the system response to the sun locational changes.
\end{abstract}

Keywords: tracking system, solar tracker, photovoltaic, optical resistaor, solar panels, operational amplifiers

\section{Introduction}

The discovery of photovoltaic phenomenon is attributed to the French physicist, A. E. Becquerel. In 1839, Becquerel observed that the battery voltage is increased when its' silver plates are placed under the sunlight. But for the first time, W. G. Adams and R. E. Day, scientists from Cambridge in 1877 , reported to the Royal Society about the photovoltaic phenomenon in a solid material in form of an article. This article included the changes in electrical properties of selenium under the sunlight. [1] In 1978, A. Mouchot designed the first solar collector or conical concentrator. The mirrors inside the cone concentrated all sun's rays at a point at the center of frustum where an absorber was installed. They called this collector AXICON. [2] The initial trends of photovoltaic systems in today's form was began in 1883 when C. Fritts built a $30 \mathrm{~cm}$ cell made of selenium and gold. [3] Heating the buildings using solar energy was introduced in 1930 and achieved a significant progress in a decade. The first solar house was built in 1938 in Massachusetts, America and their progress was so rapid that it was predicted to postpone many home heating to this method around the world up to 1970 , but its' high costs and offering fossil fuels with low cost were a barrier in this way. [4] In 1954, D. Chapin and G. Pearson and C. Fuller in Bell laboratory showed solar cells based on p-n link of silicon crystal. Solar module developed based on this type of cells could obtain 5 to 6 percent efficiency which was used for space satellite applications. [5] From then until now, the researchers have been doing many activities to improve the power of the output

*Email: pirmoradian@iaukhsh.ac.ir 
obtained from these PV panels. On the other hand, some of these researches have improved the efficiency of these cells; for instance, they reached the efficiency of $32 \%$ and in total the average of 15 to $20 \%$ using the titanium oxide under standard laboratory conditions. [8]

According to the increase of awareness and concerns about pollution and global climate changes in whole the world, environmental impact assessment points to make the most use of PV panels. [7]

Solar trackers are certainly one of the most prominent point of attention of researchers in recent decades. Tracking system improved the electrical power collected by photovoltaic panels according to the time and geographical conditions from $10 \%$ to $100 \%$. [8, 9]

Installing the tracking system leads to add some costs related to installation and motorization of moving parts, control and commanding system and also maintenance costs. These costs in addition to the impact on overall system consumption should also be surely considered in tracker installation decision.

Solar trackers can be classified into three types depending on their performance:
A) Passive
B) Equipped with a processor
C) Equipped with electro optical equipment

The main idea of passive systems is the difference in solar radiation absorption between the two parts. This difference leads to fluid evaporation of one of these two parts. This evaporation causes the move of solar collector by creating imbalance between the two parts. These systems are simple and act without any electronic control or motor and therefore their accuracy is limited and down.

The second type of these trackers are controlled based on a software which calculates the sun position at any time period and handling motors. However, these systems are often used in large installations due to the high costs of this type.

The third type are electro-optical trackers. Principle of their work is based on brightness difference in the optical resistors which layout will be in a specific form. An electronic circuit command the motor according to the resistance equal to the optical resistors. This type of resistor has high accuracy, but it should be carefully installed as well. $[6,10,11]$ many scientists have done their researches on this type of tracker according to its' accuracy and its' relatively low costs.

\section{Method and system components}

\section{1 electronic circuit}

An ideal operational amplifier has the following characteristics:

- Infinite voltage gain.

- Infinite input resistance.

- Output resistance of zero.

- Infinite bandwidth.

While none of these characteristics are not fully in accordance with the actual operational amplifiers, the results obtained with the ideal model of circuits are very close to real results in low frequencies.

In practice, operational amplifier cannot contain all mentioned characteristics in ideal mode. Because the operational amplifier is created of joining several stages of transistor amplifier and inevitably has some limitation in voltage gain, input resistance, output current and so on. Although such restrictions which exist in conventional operational amplifier don't disturb the basis of the designs implemented based on ideal mode and it just face the results with approximation, but, specific operational amplifiers with high performance can be found in the market which are very close to ideal mode in some properties and can be used in special projects. For example, some operational amplifiers are built with high speed and high output 
current and great input resistance. Recognizing the limitations of a real operational amplifier not only helps to understand the performance of designed circuits deeply, but also is required for the selection of an operational amplifier suitable for a design.

This system is built with a suitable layout based on compared resistance between the two optical resistors (light depended resistor).

Considering the two operational amplifiers with the same gain (U1, U2) which do comparison, voltages of these resistors are compared with a fix reference. In this work, this fix reference was selected with practical tests of a 2.2 kilo-ohm resistive load (figure 1). Both comparators compare their rate of base voltage of non-inverter with their base voltage of inverter which is always a constant value. The non-inverter base voltage shows the amount of light hitting the light-dependent resistors and it is the rate of resistance changes caused by receiving the light in these resistors which can be observed and recorded in form of voltage changes.

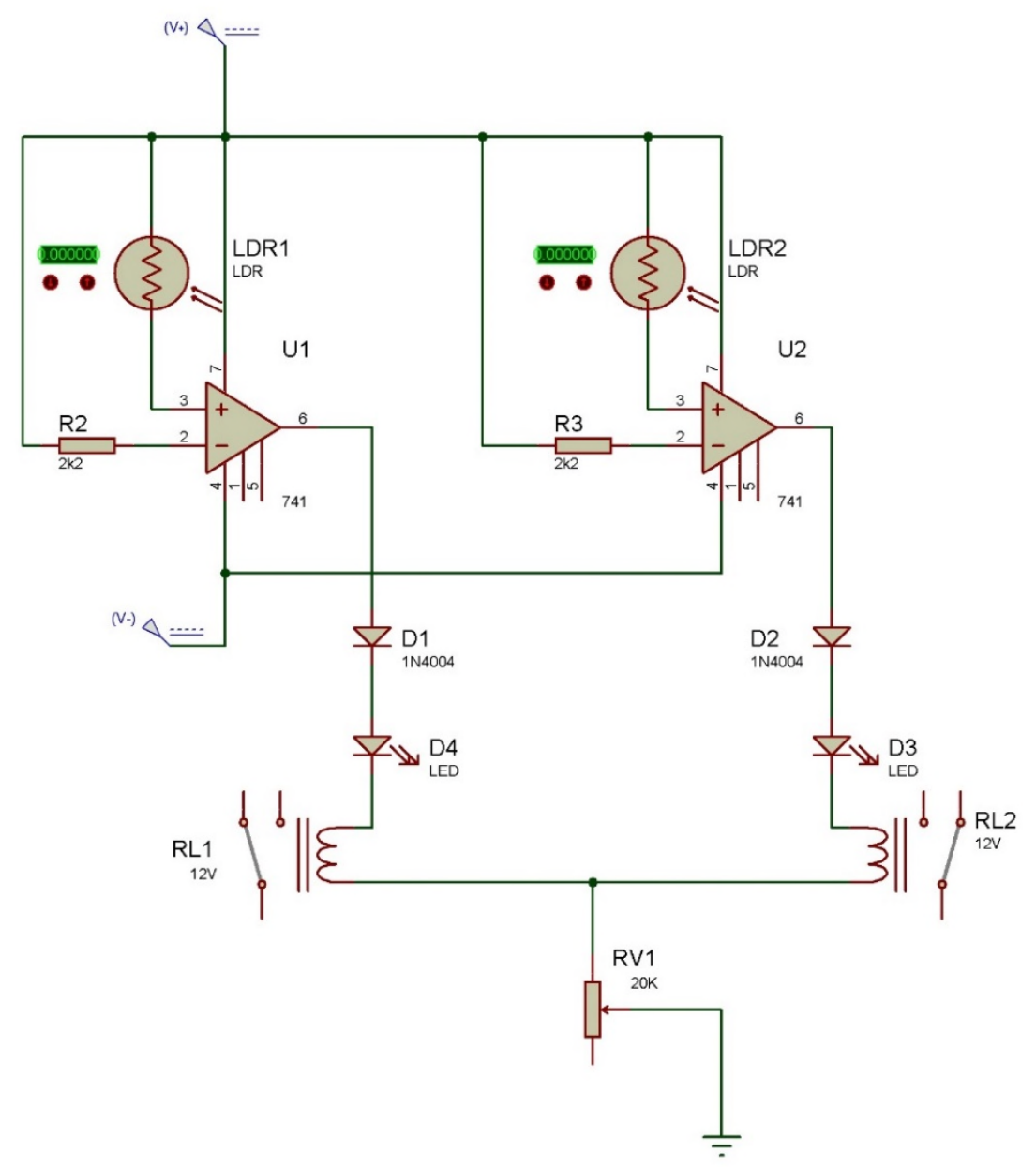

Figure 1: The electrical circuit including two similar operational amplifier 741

This circuit works on the basis of comparing the voltage for twice using two separate methods. At first, this is done via comparing the voltage of LDRs in operational amplifiers with two similar references of one type. As is understood, when we compare several values with a fix reference and achieve some results, in fact, we have compared those values with each other and getting conclusion at the end of comparison will be much easier. The first comparison in this research is done using this method.

In the second comparison, it is done by the rules of common cathode link in diodes. When two diodes are connected in form of common cathode, only one diode is located in active 
mode and this diode is the one which passes more voltage of itself. Each of diodes that is active and passes the current from itself, it also turns on the relay (electronic key) related to itself. These relays are connected to the diodes in form of series connection or the same consecutive. So in fact, a light depended resistor can controls the circuit output which receive more light.

In a very particular mode, it is possible for both parts of circuit and both diodes to be active and turn on their relays. This mode happens when the receiving light of both LDRs is the same; there is a solution in circuit layout after the relays to overcome this problem which will be discussed later.

A variable resistor has been applied to set the sensitivity of the circuit. Although this circuit has good accuracy in the test, but in order to reduce and increase the circuit accuracy, we can use other operational amplifiers layouts.

The relays have two types of output. When a relay is in passive mode, the output type one is in form of normally open (NO) circuit and the output type two is in form of normally close (NC) circuit. In active mode, the relay will be reverse which means the output type one is in form of normally close circuit and the output type two is in form of normally open circuit.

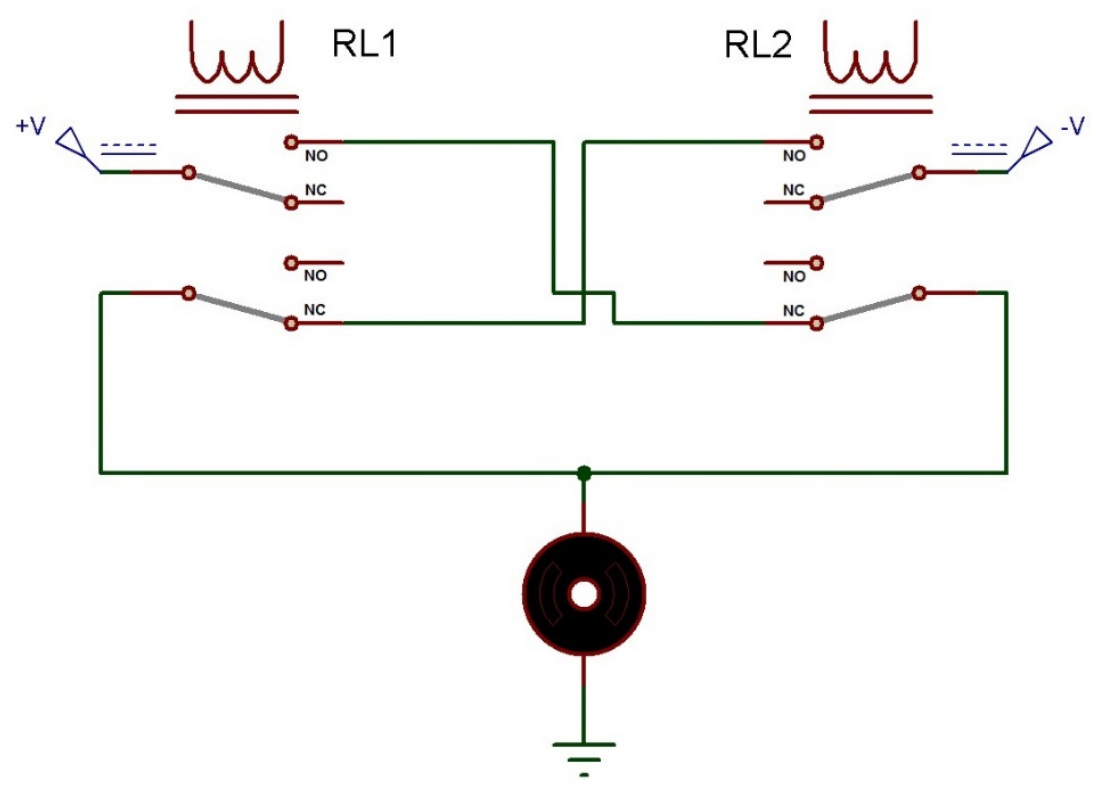

Figure 2: Circuit of relays and their connections to the motor in passive mode of circuit and relays

Relay connections to the power supply (positive and negative) and engine have been conducted in a way that if the first part of circuit is active, the motor rotates as right-handed and if the second part of circuit is active, the motor rotates as left-handed. These modes occur when the voltage of one side is more than the other side. In order to make the circuit to reach its' maximum assurance; and unifying the voltages of these parts and activating both voltage relays is not induced to the motor, bases of NC and NO relays as you see are connected (figure 2).

In this case, the electricity reach the engine when only one part of circuit is active; in cases that both parts of circuit are passive or active, the motor doesn't receive the electricity and will be in off state.

Light emitting diodes (LED) are used to see which mode is active. We can move these LEDs next to the relays to use the operating voltage of motor for turning on and show the rotation direction of motor which rotates the panel. 


\subsection{Research theory}

LDRs have a very large resistance ( 2 mega ohms in this work), this amount is reduced by receiving the light and direct sunlight. In this case, the values of special resistance for receiving the ordinary light and direct sunlight are respectively equal to 419 and $205 \mathrm{ohms}$. Special resistance of LDR can be written as following:

$$
R=R_{1}-\square R_{\text {incident }}=R_{1}-K I
$$

In which, R1 is special resistance of LDR in case of the presence of incoming released radiation and

$$
I=I_{\max } \cos \alpha
$$

In which, $\mathrm{I}_{\max }$ is direct solar radiation and $\alpha$ is the angle of the sun emitted radiation.

Figure 3 has indicated the layout of the two sensors in pyramid form. The used angles are obtained via equations 3 and 4:

$$
\begin{aligned}
& \alpha_{1}=\frac{\pi-\beta}{2}-i \\
& \alpha_{2}=\frac{\pi-\beta}{2}+i
\end{aligned}
$$

Using equations 3 and 4, the ohmic ratio will be as follows:

$$
\Omega=\frac{R_{1}-K I_{\max } \cos \alpha_{2}}{R_{1}-K I_{\text {max }} \cos \alpha_{1}}=\frac{R_{1}-K I_{\text {max }} \cos \left[\frac{\pi-\beta}{2}+\mathrm{i}\right]}{R_{1}-K I_{\text {max }} \cos \left[\frac{\pi-\beta}{2}-\mathrm{i}\right]}
$$

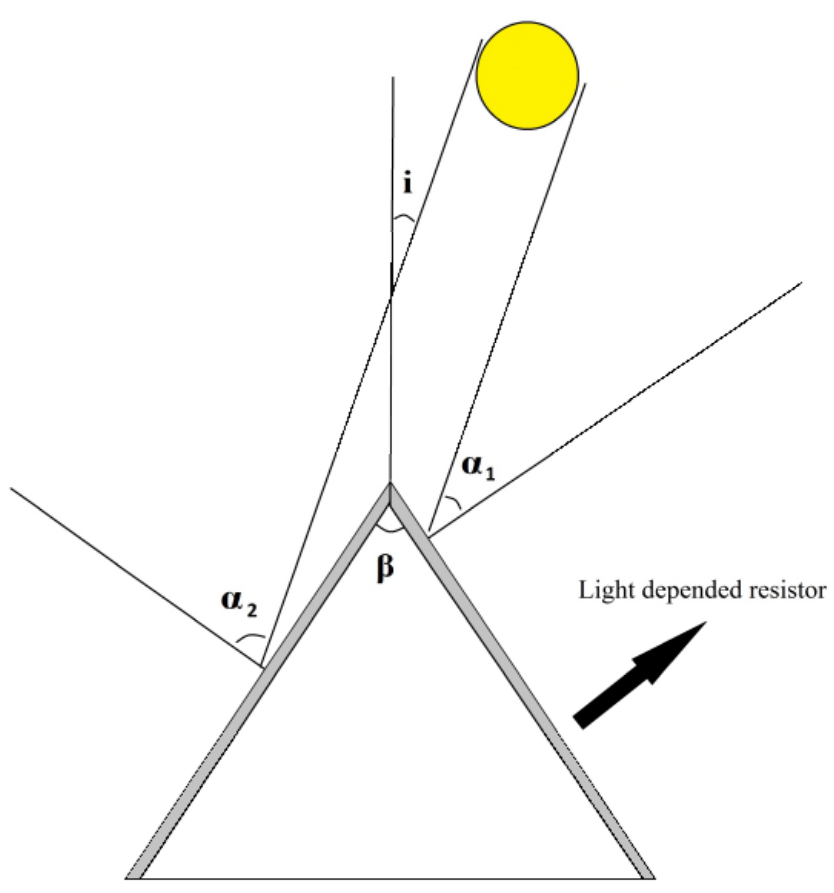

Figure 3: The sensor layout and event angle 
The curve of this equation is given in figure 4. The Excel program was used to draw this chart. The ohmic ratio of $\Omega=\frac{R_{2}}{R_{1}}$ was placed for the values of release angles relatively for the values of 0.75 and 1.33. Differences between the angles related to this event are the system errors. The written program in Excel has been implemented for several values of the angle $\beta$.

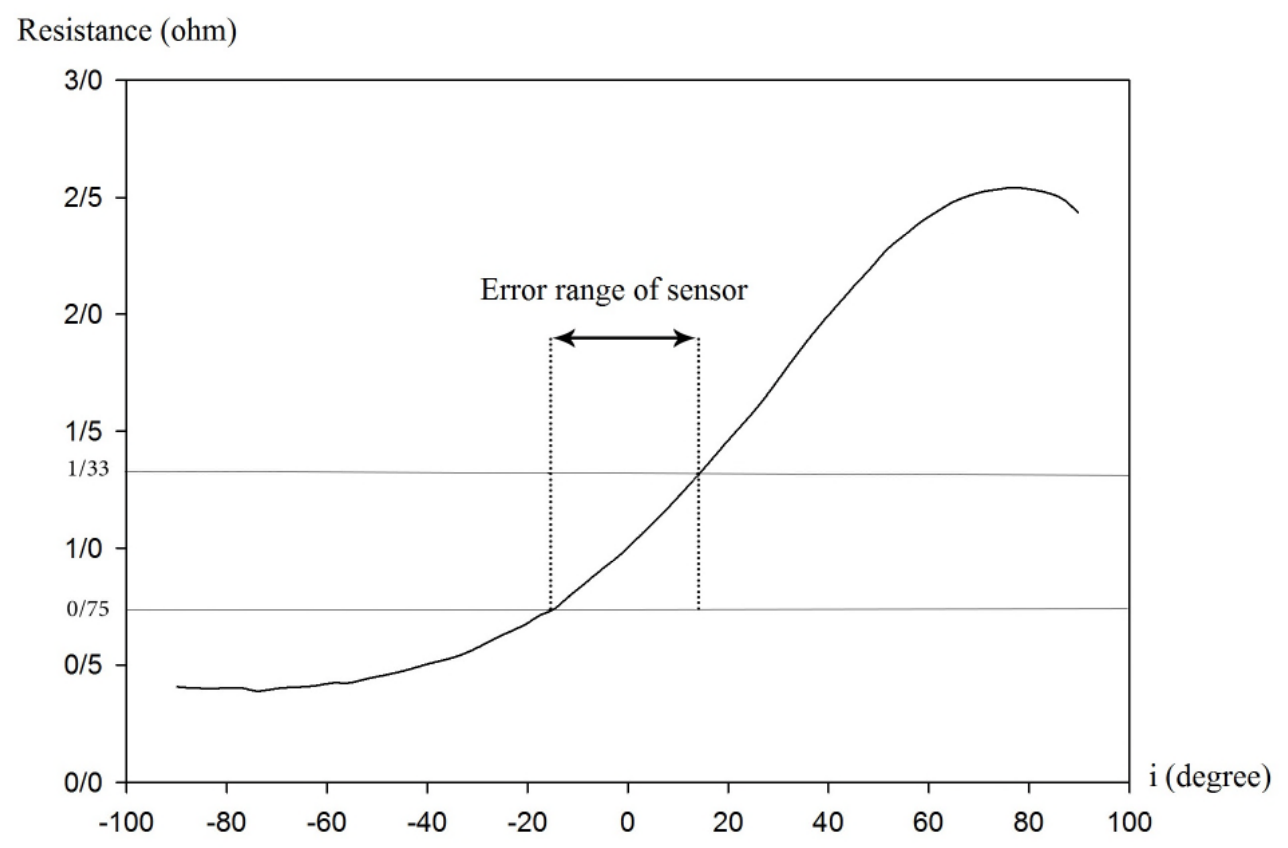

Figure 4: Numercial curve of the rate of ohmic changes

\subsection{Experience in practice}

The driving force of moving the two axes driven by two DC, $12 \mathrm{v}$ motors which control is done by two angle sensors which have been calibrated previously.

The system is configured horizontally and will be oriented to the sun by rotating the sensor motor. Various pyramid sensors $(30,60,90,120,135)$ have been investigated in order to study the effect of beta angle on the system accuracy. A special resistance of each LDR was measured with a digital multimeter for each studied angular position.

\section{Review and Results}

Special resistance used in each angle of this event is driven from the curve of the angle of sun rays and optical resistance in figure 8 . This graph indicates stable value of special resistance when the passive form of LDR is in the sun's direction. However, this $\operatorname{tr}$ i (degree) th a cosine relation when the active form is towards the sun. This reauction is in good coordination with the proposed equation in the research theory. 


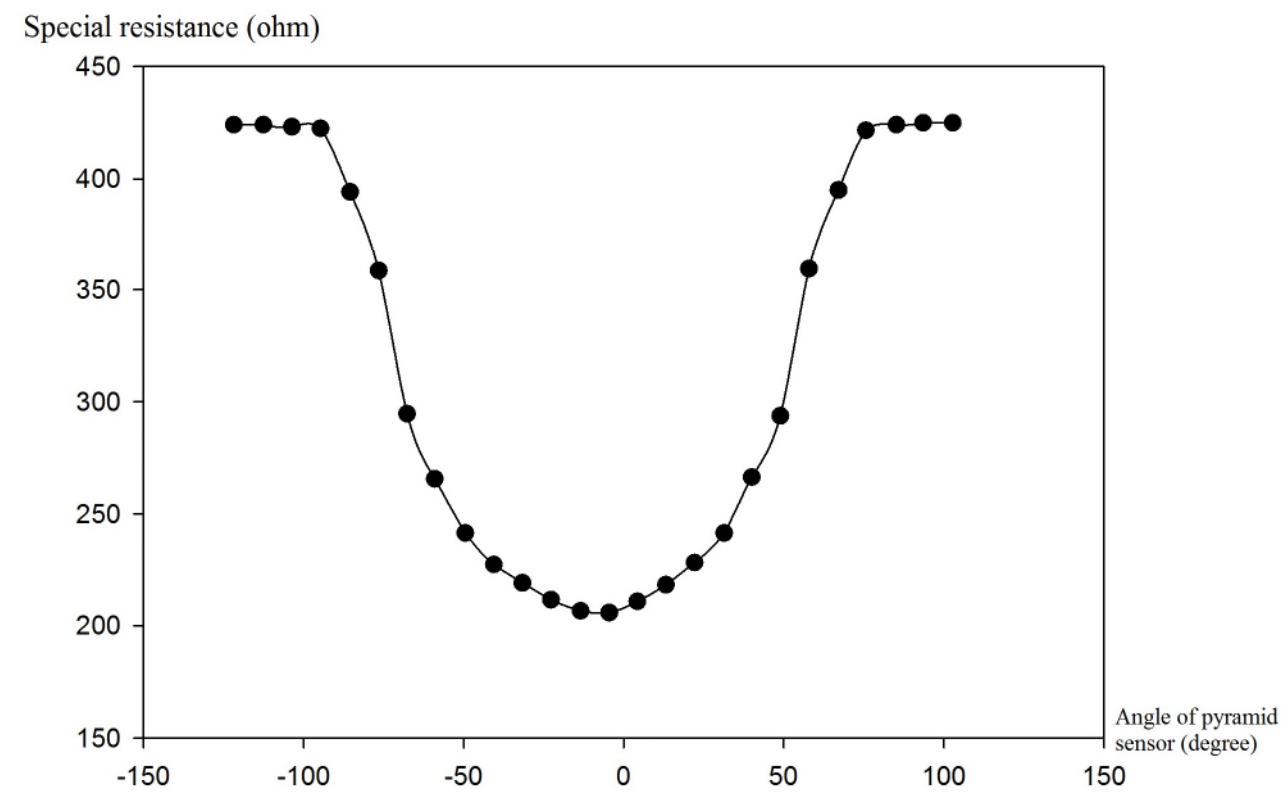

Figure 5: The characteristic curve of LDR

The ohmic ratio of special resistance is drawn in figure 6 for the studied pyramid sensors. Specific values of the electrical circuit $\Omega=0.75,1.33$ has been shown in horizontal curve in dual form. The contact of these two curves with $\Omega=f$ (i) reveals the sensor angular accuracy.
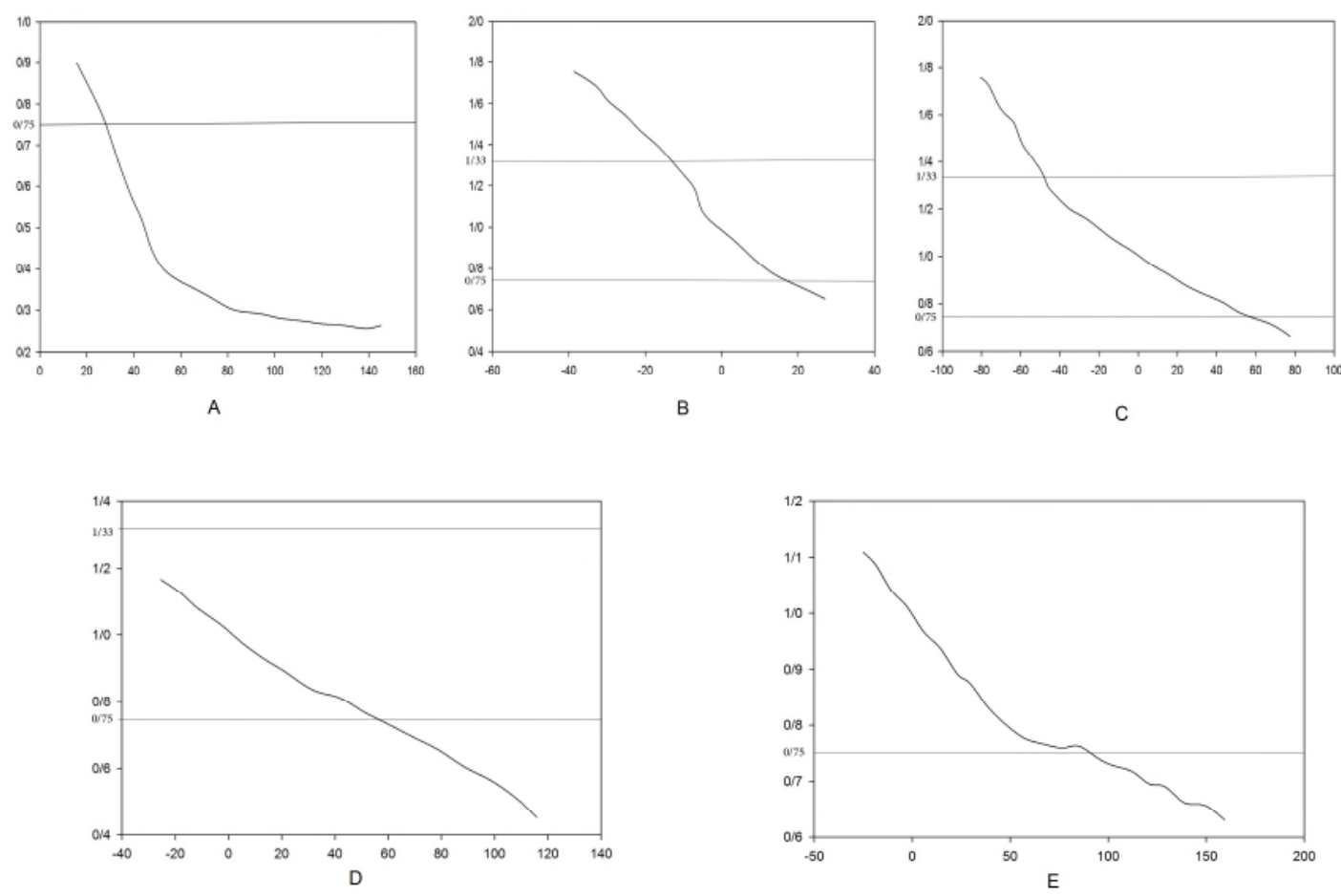

Figure 6: Rate of changes of LDRs resistance per different angles of sunlight for different angles of sensor pyramid

(A) 30 degrees angle (B) 60 degrees angle (C) 90 degrees angle (D) 120 degrees angle (E) 135 degrees angle 
Figure 7 indicates that both numerical and experimental values gave the same system error. The pyramid optimal angle for these two result categories was equal to $\beta=60$.

The system was tested in a location with 29.50720 latitude and 53.627520 longitude. Azimuth angle of the system was recorded intuitively and manually. After the setting stage (LDRs installation), the azimuth tracking system followed the sun. Motor activity rate was higher at noon and was at its greatest extent. This rate fell during the afternoon and got close to the morning rate. This process with predicted slope of the curve is very consistent with azimuth (figure 8).

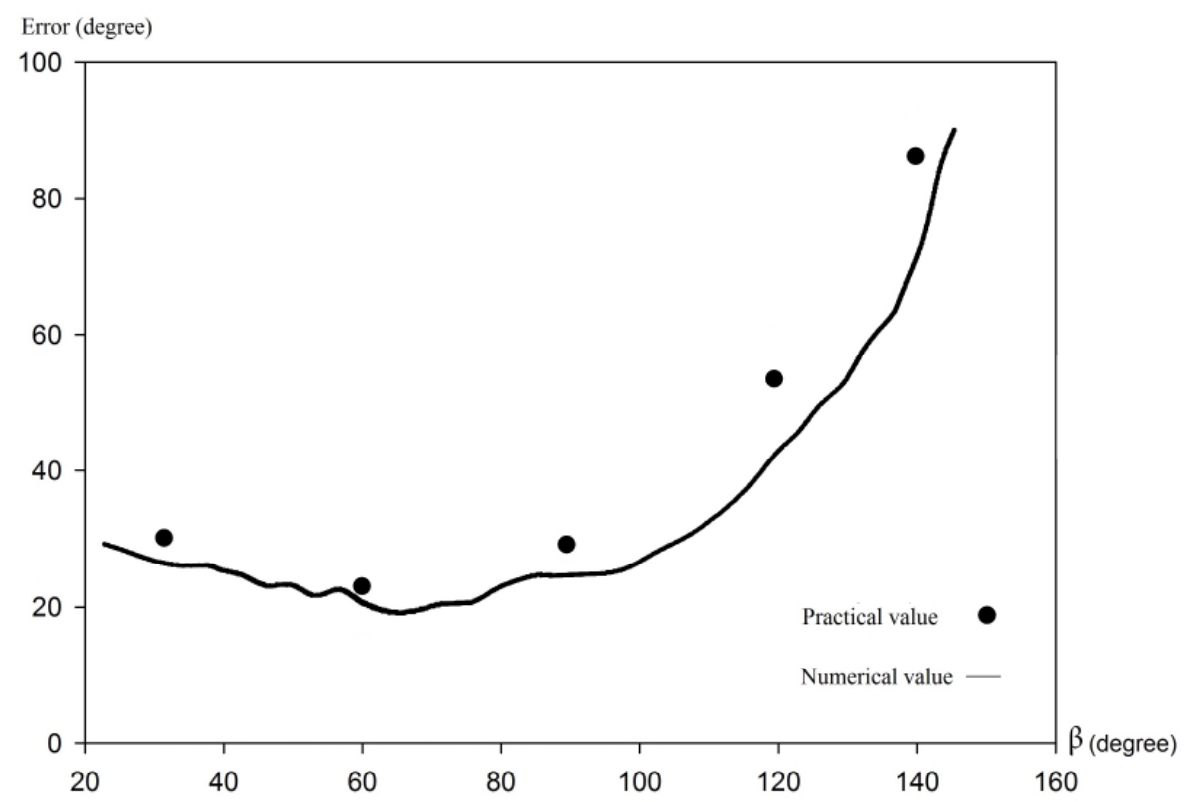

Figure 7: Numerical and practical errors for different angles

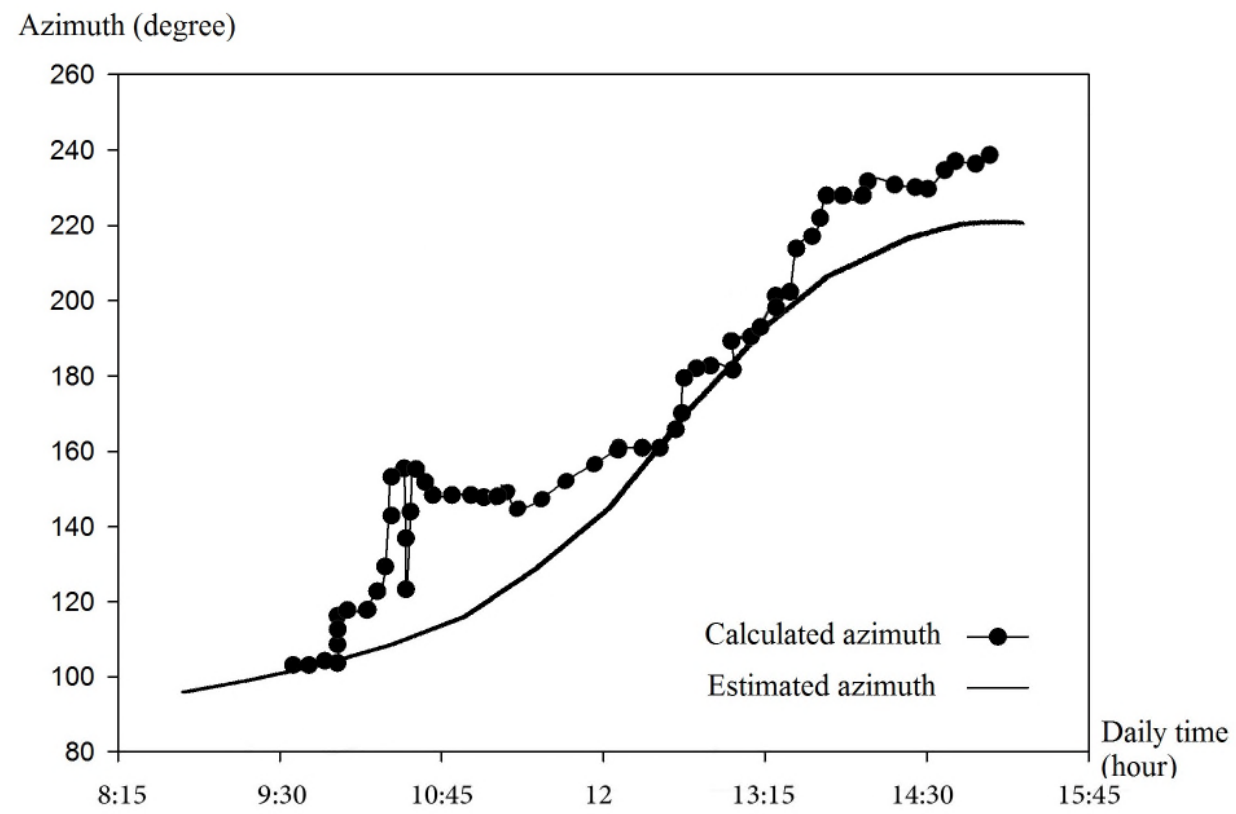

Figure 8.: The azimuths calculated practically and estimated theoretically 


\section{Conclusion}

A solar tracking system was built based on the use of two optical resistors and comparison of their voltage was evaluated by operational amplifiers. Its' accuracy is depended on the angle between two optical resistors and it is calculated based on them. The optimum angle between these two resistors was evaluated numerically and experimentally. Despite using some approximations such as linearity of used LDRs and stable amount of radiation, a significant agreement was recorded in these results. If the system is used in closed-loop mode, more accurate results can be achieved. This system is used to guide the photovoltaic panels. These results provide an acceptable solar tracking as momentarily and show that by using simple applications of operational amplifiers, we can track the sun by the best and easiest methods with high accuracy. The system accuracy can be improved using very accurate optical resistors or using optical resistors which their ohmic ratio is close to one. However, environmental parameters, such as temperature, humidity, dust, etc. can affect the accuracy of our tracking system. We can also use operational amplifiers which are professionally made for measurement. These amplifiers are closer to the ideal mode and provide a more accurate voltage difference. The electronic tools and components used in this research can be found and prepared easily in Iran.

\section{References}

[1] D. Sh. M. V. A. Bahrami, "solar cells / engineering principles, structures and technologies," p. 288, 20132013.

[2] D. A. H. Saghati, "the principles and application of solar energy," p. 327, 2001, 2011.

[3] A. Luque and S. Hegedus. (2003). Handbook of photovoltaic science and engineering .

[4] D. A. H. Saghati, "D. A. H. Aborted, "solar energy into visual narrative: Selection of solar energy activities in Iran and the world," p. 215, 2011.

[5] I. Stamatescu, I. Făgărăşan, G. Stamatescu, N. Arghira, and S. S. Iliescu, "Design and Implementation of a Solar-tracking Algorithm," Procedia Engineering, vol. 69, pp. 500-507, // 2014.

[6] J. Song, Y. Zhu, D. Xia, and Y. Yang, "A Photovoltaic Solar Tracking System with Bidirectional Sliding Axle for Building Integration," Energy Procedia, vol. 61, pp. 16381641, // 2014.

[7] S .A. S. Eldin, M. S. Abd-Elhady, and H. A. Kandil, "Feasibility of solar tracking systems for PV panels in hot and cold regions," Renewable Energy, vol. 85, pp. 228-233, 1// 2016.

[8] A. V. Arasu and T. Sornakumar, "DESIGN, DEVELOPMENT AND PERFORMANCE STUDIES OF EMBEDDED ELECTRONIC CONTROLLED ONE AXIS SOLAR TRACKING SYSTEM," Asian Journal of Control, vol. 9, pp. 163-169, 2007.

[9] M. Rahimi, M. Banybayat, Y. Tagheie, and P. Valeh-e-Sheyda, "An insight on advantage of hybrid sun-wind-tracking over sun-tracking PV system," Energy Conversion and Management, vol. 105, pp. 294-302, 11/15/ 2015.

[10] V. Zagolla, E. Tremblay, and C. Moser, "Light induced fluidic waveguide coupling," Optics Express, vol. 20, pp. A924-A931, 2012/11/05 2012.

[11] M. J. Clifford and D .Eastwood, "Design of a novel passive solar tracker," Solar Energy, vol. 77, pp. 269-280, 9// 2004. 